\title{
El aprendizaje de la Medicina en la universidad de Castilla la Mancha. Una experiencia educativa basada en el aprendizaje de las competencias profesionales.
}

\author{
Maria Teresa Alfonso-Roca
}

Directora Unidad de Educación Médica, Facultad de Medicina. Universidad de Castilla La Mancha.

\section{INTRODUCCIÓN}

La Facultad de Medicina de la UCLM creada en 1998, es la Facultad de Medicina pública más joven de España. En su creación, se estableció el compromiso, tanto por parte de los Órganos Directivos de la Universidad como de los profesores y personal de la Facultad, de desarrollar un programa innovador que capacite a los nuevos profesionales de la Medicina para dar respuesta a las necesidades de salud de la sociedad del siglo XXI.

Para ello, se desarrolló un programa educativo basado fundamentalmente en el aprendizaje y evaluación de las competencias profesionales. En Junio del 2004, se graduó la primera promoción y el pasado mes de Junio la segunda promoción formada en esta metodología docente. En estos siete años de andadura, los miembros de esta Facultad están satisfechos del enorme esfuerzo desarrollado a lo largo de este proceso. Estamos convencidos que la aplicación de los estándares internacionales de calidad en la formación de pre-grado en Medicina es el camino a seguir para dar una respuesta adecuada a las exigencias en materia de salud de nuestra sociedad.

El desarrollo de un Centro de calidad en la docencia, implica una elevada inversión para cualquier Universidad. Sin embargo, también estamos convencidos que este, no es el problema fundamental para la aplicación de estos sistemas educativos. A lo largo de esta experiencia hemos podido comprobar que en nuestra comunidad universitaria existen otros problemas de más difícil solución que el pura- mente económico. Un profesor universitario que dedica mucho tiempo a la educación o bien es un idealista o no tiene la capacidad suficiente para investigar. La promoción a puestos universitarios superiores se debe a una actuación excelente en la Investigación, y no gracias a la excelencia educativa. La actitud de los profesores frente a la educación resultará difícil de cambiar, y sobre todo de mantener, mientras persista el desequilibrio en los incentivos asociados a la educación y la investigación.

En esta ponencia describiremos el proceso seguido en la aplicación de este sistema educativo, los resultados obtenidos, así como las principales dificultades encontradas en la aplicación del mismo.

\section{EL MODELO EDUCATIVO DE LA FACULTAD DE MEDIICINA DE LA UCLM}

En la construcción del curriculum, la elección de los métodos didácticos y de su planificación raramente es considerada como un problema a tener en cuenta. La mayoría de los programas se caracterizan por un calendario de actividades docentes que normalmente no va más allá de las explicaciones orales del profesor y prácticas. Sin embargo, la planificación de los métodos educativos y de evaluación es el núcleo duro de cualquier sistema educativo. En nuestra experiencia, el diseño y planificación inicial del modelo educativo nos llevó todo el curso académico 1998/99.

El modelo educativo actual se diseña a partir del plan de estudios ${ }^{1}$, y se implanta en el curso acadé-

1. El plan de estudios de la licenciatura en Medicina de la UCLM (Plan homologado por la Comisión Académica del Consejo de Universidades en su reunión del 14 de Julio de 1998; publicado en el BOE no 239 del Martes 6 de Octubre de 1998, página 33308 y siguientes) tiene una duración de seis años y una carga lectiva total de 500 créditos de los que 185,s corresponden a créditos teóricos, 244 ,s a créditos prácticos/clínicos, 20 a asignaturas optativas (5 en el primer ciclo y 15 en el segundo ciclo) y 50 a libre configuración (25 en cada ciclo). El primer ciclo abarca las asignaturas básicas y preclínicas y el segundo ciclo se centra en las asignaturas clínicas.

2. Respecto a nuestro actual plan de estudios, las principales dificultades se centran en la excesiva carga de contenidos para el tiempo disponible en cada curso académico, y la división en algunos cursos por asignaturas. A la espera de poder llevar a cabo una modificación más profunda de nuestro actual plan de estudios, desde el curso 2002/2003, se está aplicando un sistema de incompatibilidad entre asignaturas de un curso a otro. Esto implica que un estudiante no puede superar una asignatura troncal de un curso si no tiene aprobadas las troncales del curso anterior. 
mico 1999/2000. Las necesidades para llevarlo a cabo (espacios, aulas, seminarios, recursos etc.) se van cubriendo progresivamente curso a curso.

El plan de estudios de la Licenciatura de Medicina de la UCLM se desarrolla en un modelo mixto basado en la coordinación y en algunos casos, la integración de las disciplinas, en el aprendizaje por Módulos de Objetivos y en aprendizaje por problemas, concediendo gran relevancia al desarrollo de las competencias prácticas, este programa presenta las siguientes características:

- Un aprendizaje centrado en las necesidades educativas del estudiante. El alumno participa activamente en el proceso de aprendizaje y consigue un mayor desarrollo de sus capacidades de razonamiento, de autoaprendizaje y de evaluación.

- Un aprendizaje basado en la práctica, que permite al estudiante la adquisición de las competencias clínicas y sociales que requiere el ejercicio de la profesión médica.

- Un aprendizaje multidisciplinar, que integra las ciencias básicas, clínicas y psicosociales. Durante el primer ciclo, el aprendizaje se desarrolla fundamentalmente en módulos de aprendizaje por objetivos.

\section{Módulo de Aprendizaje por Objetivos}

Un Módulo de Aprendizaje por Objetivos está formado por:

- Un máximo de SEIS asignaturas en simultáneo. Cada asignatura conlleva, a su vez, un mínimo de cuatro objetivos docentes (esta cifra es aproximada, dependiendo de la magnitud y el grado de dificultad de los objetivos que se desea alcanzar).

- Métodos específicos de aprendizaje. En función de cada uno de los distintos objetivos, se facilitan al alumno el método o métodos más adecuados para alcanzarlos, con sus procedimientos correspondientes.

- Recursos (humanos y materiales).

- Síntesis global del Módulo por objetivos y materias.

- Evaluación al finalizar cada Módulo de Aprendizaje.

\section{Estructuración pedagógica de los módulos de aprendizaje por objetivos}

Cada Módulo está formado por CINCO fases y se desarrolla a lo largo de dos o tres semanas. Cada fase presenta una metodología específica de aprendizaje.

FASE 1

Presentación y análisis de objetivos docentes

La finalidad de esta primera fase, de una dura- ción de diez a doce horas, dos horas por cada una de las asignaturas implicadas, es ayudar al alumno en el análisis y proceso que debe seguir para alcanzar el grupo de objetivos propuesto en cada una de las diferentes materias.

Esta fase presenta las siguientes ETAPAS:

1. Clarificar la problemática y cada uno de los términos enunciados en los objetivos propuestos.

2. Elaborar la lista de los distintos fenómenos o elementos a explicar.

3. Debatir y concretar los recursos de aprendizaje que se han de utilizar para cada uno de los distintos objetivos.

\section{FASE 2}

Período de autoaprendizaje

En esta fase, el alumno trabaja, de forma individual o en grupo, cada uno de los distintos objetivos propuestos en cada asignatura, siguiendo las especificaciones propuestas en la fase 1 .

Se completan además en esta fase las actividades prácticas.

\section{FASE 3}

Síntesis y actividades complementarias

En esta fase, que tiene una duración de una a tres horas por materia, los alumnos trabajan en grupos de diez o veinte alumnos, junto con el profesor de la materia. Las ETAPAS de esta fase son:

1. Puesta en común de los conocimientos adquiridos por el alumno para alcanzar cada uno de los distintos objetivos propuestos en cada bloque de las distintas materias.

2. Debatir y organizar las explicaciones propuestas, con el fin de producir una descripción coherente de los mecanismos que originan los distintos fenómenos y avanzar en su comprensión. 3. Realizar las distintas actividades complementarias de los distintos objetivos (prácticas, resolución de problemas, cuestiones ...).

\section{FASE 4}

Autoaprendizajes tutelados

Nuevo período de autoaprendizaje. En este período, el alumno tiene a su disposición al profesorado y puede solicitar cuantas tutorías o actividades complementarias considere necesarias para la total comprensión de los objetivos fijados en cada Módulo.

\section{FASE 5}

Evaluación

Al finalizar cada Módulo de objetivos, y de forma 
sistemática, se lleva a cabo una evaluación conjunta del mismo.

En cada Módulo, el alumno dispone de los recursos que se consideran básicos para alcanzar los distintos objetivos. Además, dispone de una oferta de actividades prácticas complementarias con tutela del profesor.

\section{Metodologías pedagógicas de aprendizaje en las materias básicas.}

La docencia en grupos pequeños (20 estudiantes en las preclínicas) y por Módulos de Objetivos permite una mayor proximidad profesor-alumno en cada una de las fases de aprendizaje. Esta mayor proximidad facilita en principio una participación más intensa del estudiante. Sin embargo, éste se muestra en muchas ocasiones reticente a participar y a compartir con sus compañeros los análisis que pueda haber efectuado o las cuestiones que pueda tener. Por esta razón, la Facultad de Medicina de la UCLM, mediante la Unidad de Educación Médica, organiza seminarios para el profesorado encaminados a desarrollar y poner en practica técnicas de intervención pedagógica para incrementar la participación del estudiante en su autoaprendizaje.

La experiencia acumulada estos años por los profesores de las diversas materias nos permite hoy obtener el mayor provecho de cada una de las fases de aprendizaje y conseguir que los estudiantes sean capaces de perder sus miedos a la participación en grupo y al trabajo en equipo, que tan importante y necesario será en la práctica de su profesión.

\section{Metodologías pedagógicas de aprendizaje en el medio clínico.}

En el mes de febrero del 2001, se inició el aprendizaje en el medio clínico. La planificación desarrollada para el aprendizaje de las materias clínicas introduce el aprendizaje por problemas (APP), talleres de habilidades clínicas, seminarios, aprendizaje con pacientes simulados y la evaluación de las competencias clínicas a través de la OSCE (Objecfive Structured Clinical Examination). La estructura del aprendizaje en el medio clínico presenta diferencias respecto al aprendizaje por módulos de objetivos: La estructuración del aprendizaje es por rotación, (cada grupo está formado por un máximo de 10 estudiantes) en los distintos servicios clínicos implicados, y por semanas. Las rotaciones tienen una duración de entre cuatro y doce semanas, dependiendo de los objetivos del aprendizaje especificados en cada una de ellas. Al finalizar cada rotación, se lleva a cabo una evaluación de la misma. En las rotaciones de mayor duración, se llevan a cabo varias evaluaciones. Cada una de estas evaluaciones comprende una parte cognitiva y una parte de habilidades y actitudes.

Cada semana comprende un total de treinta y cinco horas de actividades distribuidas de la siguiente forma:

Veinticinco horas de aprendizaje práctico en los distintos servicios especificados en la rotación.

Diez horas de actividades en aula. Estas actividades comprenden:

Cuatro horas de seminarios APP

Cuatro horas de talleres

Dos horas de seminarios de síntesis.

Al finalizar la rotación, se llevan a cabo los exámenes que tiene una duración aproximada de entre dos y seis horas, dependiendo del tipo de pruebas. $\mathrm{Al}$ finalizar el curso, se lleva a cabo una evaluación final de las competencias clínicas. La superación de esta prueba es imprescindible para aprobar el curso académico.

\section{Evaluación de Las Competencias Clínicas}

La Facultad de Medicina de la UCLM, es la primera Facultad de Medicina en España en llevar a cabo de forma sistemática, al finalizar cada curso del segundo ciclo de formación, un examen clínico objetivo estructurado sobre las competencias clínicas que el estudiante ha alcanzado durante ese año. La evaluación de las competencias profesionales constituye el elemento central en el desarrollo de las profesiones, siendo la clave para garantizar la calidad de los servicios sanitarios.

La mayor parte de las pruebas de evaluación de estudiantes de Medicina utilizadas en las universidades españolas exploran aspectos predominantemente cognoscitivos, dejando a un lado la valoración de la competencia clínica. El resultado de este modo de evaluar es que, tras la licenciatura, muchos estudiantes son incapaces de identificar los problemas de salud relevantes en los pacientes, tomar decisiones (propuestas de acción) y ejecutarlas adecuadamente. La competencia profesional de un médico y la buena práctica médica no se vinculan sólo a la memorización, sino sobre todo al razonamiento clínico, a la toma de decisiones, a la resolución de problemas y a las habilidades en las relaciones interpersonales. Es en este sentido en el que la Facultad de Medicina de la UCLM ha introducido nuevas técnicas de evaluación que exploran objeti- 
vamente todas estas áreas de competencia que un médico debe adquirir en cada nivel de su formación.

La técnica de evaluación utilizada en esta Facultad de Medicina es el examen clínico objetivo estructurado, OSCE (Objective Structured Clinical Examination). Este es un sistema para la evaluación de competencias clínicas en el que dichas competencias, especialmente las actitudes y las habilidades, se evalúan de forma planificada y estructurada, con especial atención dedicada a la objetividad del examen.

Este tipo de pruebas tiene el potencial de evaluar un amplio rango de conocimientos y habilidades clínicas en un número determinado de estudiantes durante un solo período de examen. Su estructura básica es un circuito de estaciones de evaluación. En cada estación, el estudiante se enfrenta a una situación diseñada para evaluar competencias clínicas y conocimientos médicos específicos. Los estudiantes rotan en forma sucesiva y simultánea a través de las estaciones con intervalos de igual duración.

La objetividad de este método de evaluación es alta, ya que cada estudiante es expuesto a la misma situación simulada, con o sin pacientes estandarizados, y es examinado por el mismo evaluador, quien aplica criterios estándares prediseñados (plantillas de observación) iguales para todos los estudiantes.

\section{RESULTADOS}

Durante el curso académico 2000/2001, se inicia el proceso de elaboración de este tipo de exámenes. En esta primera ocasión solo se desarrolla una evaluación de las competencias clínicas en la asignatura de Semiología y Propedéutica. Este examen fue realizado por 78 estudiantes al finalizar su tercer curso de formación.

La OSCE de $4^{\circ}$ curso consta de:

El primer examen OSCE, se desarrolla al finalizar el curso académico 2001/2002 a los estudiantes de $4^{\circ}$ curso. Un total de 75 estudiantes realizaron durante 3 días dichas pruebas.

Un total de 25 estaciones configuraron esta OSCE, distribuidas de la siguiente forma:

8 casos clínicos con pacientes estandarizados

(simulados)

3 estaciones clínicas de dermatología.
4 estaciones de casos de psiquiatría

8 estaciones de ginecología

2 estaciones de hematología.

A partir de este curso académico (2001/2002), los exámenes OSCE, se sistematizan y se llevan a cabo al finalizar todos los cursos clínicos.

La OSCE de 3er curso consta de:

8 estaciones de Pacientes Estandarizados

(simulados)

1 estación de Radiología

1 estación de Microbiología

1 Estación de Anatomía Patológica

1 Estación de habilidades quirúrgicas.

La OSCE de $5^{\circ}$ consta de:

8 casos clínicos con pacientes simulados

1 estación de oftalmología

2 estaciones de pediatría

La OSCE de $6^{\circ}$ consta de:

8 casos clínicos con pacientes estandarizados

1 estación de reanimación (soporte vital

básico/avanzado).

\section{OTROS DATOS}

Para cada uno de los cursos se invierte una media de 250 horas de preparación (incluido el tiempo de entrenamiento de los pacientes estandarizados)

Actualmente disponemos de 60 pacientes simulados que participan en el conjunto de todas las pruebas OSCE que se realizan de 30/30 a 60/60 en la facultad de medicina.

En cada curso los estudiantes realizan dicha prueba a lo largo de 3 días. El tiempo medio de realización de la prueba es de 3 horas por estudiante.

Los resultados obtenidos por los estudiantes en estas pruebas suelen coincidir con las calificaciones obtenidas en las evaluaciones de las competencias clínicas que realizan en cada rotación. Sin embargo, no se observa una correlación igual con los resultados obtenidos en los exámenes test de conocimientos. Es decir los estudiantes que obtienen una mayor puntuación en los exámenes test, suelen obtener una puntuación menor en este examen de competencias clínicas. 